\title{
Inhibition of Al-2 Quorum Sensing and Biofilm Formation in Campylobacter jejuni by Decanoic and Lauric Acids
}

\author{
Shenmiao Li,1,2, Kelvin Ka-wan Chan', Marti Z. Hua',2, Greta Gölz³ and Xiaonan Lu',2* \\ ${ }^{1}$ Food, Nutrition and Health Program, Faculty of Land and Food Systems, The University of British Columbia, Vancouver, \\ BC, Canada, ${ }^{2}$ Department of Food Science and Agricultural Chemistry, Faculty of Agricultural and Environmental Sciences, \\ McGill University, Montreal, QC, Canada, ${ }^{3}$ Institute of Food Safety and Food Hygiene, Freie Universität Berlin, Berlin, \\ Germany
}

\section{OPEN ACCESS}

Edited by:

Haifeng Zhao,

South China University of Technology,

China

Reviewed by:

Abhinav Upadhyay,

University of Connecticut,

United States

Hyunjoon Park,

Handong Global University,

South Korea

${ }^{*}$ Correspondence:

Xiaonan Lu

xiaonan.lu@mcgill.ca

Specialty section:

This article was submitted to

Food Microbiology,

a section of the journal

Frontiers in Microbiology

Received: 08 November 2021

Accepted: 22 December 2021

Published: 13 January 2022

Citation:

Li S, Chan KK-w, Hua MZ, Gölz G and LuX (2022) Inhibition of Al-2

Quorum Sensing and Biofilm

Formation in Campylobacter jejuni by

Decanoic and Lauric Acids.

Front. Microbiol. 12:811506.

doi: 10.3389/fmicb.2021.811506
Campylobacter jejuni is a major bacterial cause of human diarrheal diseases worldwide. Despite its sensitivity to environmental stresses, C. jejuni ubiquitously distributes throughout poultry production chains. Biofilm formation mediated by quorum sensing is suggested to be critical to the survival of $C$. jejuni in agroecosystem. C. jejuni possesses LuxS, the enzyme involved in the production of autoinducer-2 (Al-2) signaling molecules. In this study, two fatty acids, namely decanoic acid and lauric acid, were identified to be effective in inhibiting Al-2 activity of $C$. jejuni. Both decanoic acid and lauric acid at 100 ppm inhibited $\sim 90 \%$ Al-2 activity $(P<0.05)$ of $C$. jejuni without bacterial inactivation. The biofilm biomass of two $C$. jejuni strains was reduced by $10-50 \%$ $(P<0.05)$ after treatment by both fatty acids, while increased biofilm formation was observed for one $C$. jejuni strain. In addition, both fatty acids effectively reduced the motility of all tested $C$. jejuni strains. These findings can aid in developing alternative C. jejuni control strategies in agri-food and clinical settings.

Keywords: Campylobacter jejuni, quorum sensing (QS), biofilm, motility, fatty acids

\section{INTRODUCTION}

Campylobacter is recognized as a major bacterial cause of human diarrheal diseases worldwide (Kaakoush et al., 2015). In 2010, Campylobacter infections and the post-infectious sequelae caused an estimated 166 million diarrheal illnesses and 37,600 deaths worldwide, resulting in significant socio-economic implications (Kirk et al., 2015). Epidemiological evidence collected from FoodNet indicated that Campylobacter outnumbers Salmonella to become the most common bacterial agent causing human diarrheal disease since 2017 in the United States (Marder et al., 2017). Moreover, the incidence of Campylobacter infections increased by 13\% in 2019 compared to 2016-2018 (Tack et al., 2020). Among the identified species, Campylobacter jejuni is the predominant cause of human infections (World Health Organization, 2013). C. jejuni is considered a commensal bacterium in poultry that inhabits the intestine and can be spread to agri-food related environments at different segments, such as chicken farms, slaughterhouses, and processing plants (Sahin et al., 2015). Although C. jejuni is nutritionally fastidious and sensitive to food production-associated stresses, its adaptability allows it to survive and remain infectious in various environmental conditions 
(García-Sánchez et al., 2018). The most common transmission of $C$. jejuni to humans is attributed to handling and consuming contaminated animal products (Wagenaar et al., 2013).

C. jejuni continues to pose a significant burden to public health while tremendous efforts have been put to reduce Campylobacter-associated foodborne illnesses. In the early decades, antibiotics (e.g., fluoroquinolone and macrolide) were widely used as therapeutic or prophylactic drugs in human medicine and poultry production (Tang et al., 2017). Antibiotic feed additives is a cost-effective solution to increase poultry production by improving growth performance and suppressing bacterial infectious in a short time (M'Sadeq et al., 2015). Nevertheless, the emergence of antibiotic-resistant C. jejuni has been observed following the extensive use of antibiotics in broiler breeder farms (Guo et al., 2018; Mehdi et al., 2018). These antibiotic-resistant C. jejuni strains threaten the effectiveness of those commonly used antibiotics in treating human infections (Centers for Disease Control Prevention, 2019). Since an EUwide ban on antimicrobial growth promoters in 2006, many other countries have phased out medically important antibiotic use in animal agriculture (Martin et al., 2015). In addition, stricter regulations of Campylobacter in poultry production have been implemented to control the incidence of C. jejuni infections. In 2016, the US Department of Agriculture's Food Safety and Inspection Service updated food safety standards with lower tolerance of Campylobacter in raw poultry products so as to reduce campylobacteriosis (Us Department of Agriculture Food Safety and Inspection Service, 2016). Therefore, developing effective alternative control measures to reduce the prevalence of C. jejuni in the agroecosystem is highly necessary.

The intercellular communication system of bacteria, namely quorum sensing, is a potential target for pathogen control. Quorum sensing enables bacteria to coordinate their physiological functions to adapt to population changes, collectively act as a group, and mediates behaviors including expression of virulence factors, change of nutrient acquisition, production of public goods, and biofilm formation (Papenfort and Bassler, 2016). To orchestrate population-wide behaviors, bacteria communicate via different types of self-produced chemical signals called autoinducers. Most of the identified autoinducers in the early studies can be classified into three categories, namely $N$-acyl-homoserine lactones (AHLs), autoinducing peptides (AIPs), and autoinducer-2 (AI-2). AHLs, AIPs, are used by Gram-negative bacteria, Gram-positive bacteria, respectively (Reading and Sperandio, 2006). Besides, AI-2 as a type of universal signaling molecules are utilized by both Gram-negative and Gram-positive bacteria (Reading and Sperandio, 2006). Through quorum sensing, bacteria can take a census of surrounding cells, recognize if they are kin or not, and respond to the signals in a coordinated fashion contingent on the type and concentration of quorum sensing molecules (Papenfort and Bassler, 2016). Many studies demonstrated the great potential of quorum sensing inhibitors in controlling bacterial infections (Brackman et al., 2011; Jiang and Li, 2013; Jiang et al., 2019). By preventing the phenotypes that enhance adaption, survival, and virulence, quorum sensing inhibitors increase pathogens' susceptibility to antimicrobials and physical treatments and pathogenicity (Bettenworth et al., 2019). In C. jejuni, cell density-dependent phenotypes (e.g., motility, host colonization, virulence, and biofilm formation) are associated with AI-2-mediated quorum sensing system (Plummer, 2012). S-ribosylhomocysteinase, also known as LuxS, produces AI-2 as a by-product during methionine recycling (Schauder et al., 2001), and LuxS coding gene has been identified in C. jejuni (Parkhill et al., 2000). AI-2 production in luxS-deficient $C$. jejuni mutant is completely repressed (Plummer et al., 2011). With the abolished AI-2 activity, luxS-deficient C. jejuni also showed weakened cooperative behaviors, including autoagglutination (Jeon et al., 2003), motility (Plummer et al., 2011), host colonization and biofilm formation (Quiñones et al., 2009), all of which are key factors affecting the survival of $C$. jejuni. Therefore, inhibiting quorum sensing in C. jejuni could be a novel approach to control this microbe in agri-food and clinical settings.

Bactericidal effects of a wide variety of phytochemicals and fatty acids, sterols, and glycerols (e.g., cinnamaldehyde, myristic acid, $\beta$-sitosterol, monomyristin, etc.) have been widely studied due to their low cost and high safety (Barbieri et al., 2017). Some short-chain and medium-chain fatty acids can be used as sanitizers for food contact surfaces with $\mathrm{pH}<4.0$ (Marriott et al., 2006). Nevertheless, carboxylic acid at high concentration and low $\mathrm{pH}$ are corrosive to equipment, and long-term use of acidic solution may create niches for biofilm development due to equipment tear and wear (Schmidt, 1997). A few studies reported potential AI-2 inhibitory effects of long-chain fatty acids, including linoleic acid, oleic acid, palmitic acid, and stearic acid (Widmer et al., 2007). However, the potential of quorum sensing inhibition by fatty acids at low concentrations without bactericidal effect has not been investigated yet. In this study, 12 natural-origin compounds on C. jejuni AI-2 activity were investigated, the chemical structure and name of each compound were listed in Supplementary Table 1. Two medium-chain fatty acids were identified to be effective on quenching AI-2 of C. jejuni at their subinhibitory concentration. Their effects on biofilm formation and motility were also evaluated.

\section{MATERIALS AND METHODS}

\section{Chemicals and Bacterial Strains}

Hexanoic acid (> 98\% purity), octanoic acid (> 98\% purity), and decanoic acid (> 99\% purity) were purchased from Alfa Aesar (Ward Hill, MA, United States). Lauric acid was obtained from Acros Organics (Fair Lawn, NJ, United States). 4-Hydroxy-2,5-dimethyl-3(2H)-furanone $(\geq 98 \%$ purity), 5-Ethyl-4-hydroxy-2-methyl-3(2H)-furanone ( $\geq 96 \%$ purity), 2,5-Dimethyl-3-oxo-(2H)-fur-4-yl butyrate ( $\geq 93 \%$ purity), crotonic acid ( $\geq 98 \%$ purity), trans-ferulic acid ( $\geq 99 \%$ purity), quercetin ( $\geq 95 \%$ purity), trans-cinnamaldehyde ( $\geq 99 \%$ purity), and naringenin ( $\geq 95 \%$ purity) were obtained from Sigma-Aldrich (Oakville, ON, Canada). The stock solutions of hexanoic acid, octanoic acid, decanoic acid, lauric acid, 4-hydroxy-2,5-dimethyl-3(2H)-furanone, 5-ethyl-4-hydroxy2-methyl-2(2H)-furanone, 2,5-dimethyl-3-oxo-2(H)-fur-4-yl butyrate, crotonic acid, and trans-ferulic acid were prepared 
by separately dissolving each compound in absolute ethanol to achieve a concentration of 20,000 ppm. The stock solution of each compound was further diluted to working solutions as 400 , $300,200,150,100,50$, and $25 \mathrm{ppm}$ with $\mathrm{MH}$ broth upon the test. The stock solutions of quercetin, trans-cinnamaldehyde, and naringenin were prepared by separately dissolving each compound in absolute ethanol to achieve a concentration of $5,000 \mathrm{ppm}$. The stock solution of each compound was further diluted to working solutions as $100,50,25,12.5,6.25$, and $3.125 \mathrm{ppm}$ with $\mathrm{MH}$ broth upon the test. $\mathrm{MH}$ broth with 1 and $0.5 \%$ ethanol were used as the control groups. We verified that the addition of ethanol at a concentration of $1 \%$ or below did not affect $C$. jejuni cell viability and growth (data not shown).

Three C. jejuni strains, namely F38011 (human clinical isolate), NCTC11168 (human clinical isolate), and ATCC33560 (bovine feces isolate), were routinely cultivated either on Mueller Hinton II agar (BD $\mathrm{BBL}^{\mathrm{TM}}$ ) plates supplemented with 5\% defibrinated sheep blood (MHB agar) or in $\mathrm{MH}$ broth (BD Difco $\left.{ }^{\mathrm{TM}}\right)$ at $37^{\circ} \mathrm{C}$ under microaerobic condition $\left(85 \% \mathrm{~N}_{2}, 10 \%\right.$ $\mathrm{CO}_{2}, 5 \% \mathrm{O}_{2}$ ) with constant shaking at $175 \mathrm{rpm}$. Vibrio harveyi strains $\mathrm{BB} 152$ (AI-2 positive control) and BB170 (AI-2 reporter) were cultivated either on Marine agar 2216 (BD Difco ${ }^{\mathrm{TM}}$ ) plates or in autoinducer bioassay (AB) medium (Greenberg et al., 1979) prepared in the lab that contains $0.33 \mathrm{~mol} \cdot \mathrm{L}^{-1} \mathrm{NaCl}$, $0.05 \mathrm{~mol} \cdot \mathrm{L}^{-1} \mathrm{MgSO}_{4}, 0.2 \%(\mathrm{w} / \mathrm{v})$ vitamin-free casamino acids, $1 \%(\mathrm{v} / \mathrm{v})$ glycerol, $1 \mathrm{mmol} \cdot \mathrm{L}^{-1} \mathrm{~L}$-arginine (filtration sterilized), and $10 \mathrm{mmol} \cdot \mathrm{l}^{-1}$ potassium phosphate $\left(\mathrm{pH} \mathrm{7.0)}\right.$ at $30^{\circ} \mathrm{C}$ under aerobic condition with constant shaking at $175 \mathrm{rpm}$.

\section{Determination of Subinhibitory Concentrations}

The highest subinhibitory concentration of all the selected compounds was determined using a microtiter broth dilution method, as described in a previous study (Duarte et al., 2016). In brief, overnight $C$. jejuni culture of each strain was diluted to $\sim 10^{8} \mathrm{CFU} / \mathrm{mL}$ in $\mathrm{MH}$ broth. One hundred microliters of the working solution of each compound were separately added to each well of a sterile 96-well polystyrene plate (Corning, untreated, flat-bottom), followed by inoculation of $100 \mu \mathrm{L}$ of C. jejuni culture $\left(\sim 10^{8} \mathrm{CFU} / \mathrm{mL}\right)$. Each strain that cultivated in $\mathrm{MH}$ broth without addition of each compound served as positive control. Blank controls were performed using sterile $\mathrm{MH}$ broth supplemented with the working solution of each compound at the highest concentration. Then, the inoculated 96-well plates were covered by low evaporation lids and incubated at $37^{\circ} \mathrm{C}$ under microaerobic condition for $48 \mathrm{~h}$. The highest concentration of the compound that did not inhibit the visible growth of $C$. jejuni culture (compared to positive control) was taken as the subinhibitory concentration for the strain.

\section{Screening of Al-2 Inhibitors \\ Preparation of Cell-Free Supernatants}

Quantification of AI-2 activity was performed via $V$. harveyi AI2 assay was conducted following the protocol described in a previous study with minor modifications (Bassler et al., 1997).
The preliminary data showed that $C$. jejuni cultivated in a static condition in a 96-well plate has a higher AI-2 activity at $48 \mathrm{~h}$ than that either at 24 or $72 \mathrm{~h}$ (Supplementary Figure 1). In addition, C. jejuni ATCC 33560 forms relatively less biofilm than other strains. A larger portion of AI-2 molecules was identified in biofilm supernatant than biofilm of ATCC 33560 (Supplementary Figure 2). Based on these preliminary results, thereby we selected C. jejuni ATCC 33560 as the representative strain and $48 \mathrm{~h}$ as the treatment time for AI-2 inhibitor screening.

Quorum sensing inhibition capabilities of 12 compounds at the subinhibitory concentrations against C. jejuni ATCC 33560 cells were evaluated at the concentration summarized in Table 1. One hundred microliters of the working solution of each compound were added to each well of sterile 96-well polystyrene plates, followed by inoculation of $100 \mu \mathrm{L}$ of C. jejuni ATCC 33560 overnight culture $\left(\sim 10^{8} \mathrm{CFU} / \mathrm{mL}\right)$. The treatment solutions were replaced with sterile $\mathrm{MH}$ broth and $\mathrm{MH}$ broth with $1 \%$ ethanol for the broth control and ethanol control. Then, the microtiter plates were covered with low evaporation lids and incubated at $37^{\circ} \mathrm{C}$ under the microaerobic condition for $48 \mathrm{~h}$. The supernatant from each well was collected separately after incubation and then centrifuged at $8,000 \times g$ for $10 \mathrm{~min}$ and passed through 0.22 $\mu \mathrm{m}$ PES filters. The collected cell-free supernatants (CFSs) were stored at $-20^{\circ} \mathrm{C}$ until further usage.

\section{Vibrio harveyi Autoinducer-2 Bioassay}

To detect the AI-2 activity in each collected CFSs collected in section "Preparation of Cell-Free Supernatants." The reporter strain $V$. harveyi $\mathrm{BB} 170$ and the positive control strain $V$. harveyi $\mathrm{BB} 152$ were grown in $\mathrm{AB}$ medium for $16 \mathrm{~h}$ at $30^{\circ} \mathrm{C}$ under the aerobic condition with constant shaking at $175 \mathrm{rpm}$. The $V$. harveyi BB170 was then diluted 5,000-fold with fresh $\mathrm{AB}$ medium. The CFSs $V$. harveyi BB152 were obtained by centrifuging the culture supernatant at $8,000 \times g$ for $10 \mathrm{~min}$ and passed through $0.22-\mu \mathrm{m}$ PES filters. One hundred and eighty microliters of diluted $V$. harveyi BB170 culture were added to each well of a sterile white 96-well plate (opaque, flat bottom). Twenty microliters of collected C. jejuni and V. harveyi BB152 CFSs were separately added to each well containing diluted

TABLE 1 | Concentration of each compound used in the screening of quorum sensing inhibitory effects.

Compound Concentration (ppm)

Hexanoic acid 200

Octanoic acid

100

Decanoic acid

100

Lauric acid

100

4-Hydroxy-2,5-dimethyl-3(2H)-furanone

200

5-Ethyl-4-hydroxy-2-methyl-2(2H)-furanone

200

2,5-Dimethyl-3-oxo-2(H)-fur-4-yl butyrate

200

Crotonic acid

200

Trans-Ferulic acid

200

Quercetin

25

Trans-cinnamaldehyde

12.5

Naringenin

25 
reporter suspension. One hundred and eighty microliters of diluted reporter suspensions with $20 \mu \mathrm{L}$ of uninoculated $\mathrm{AB}$ medium were served as the negative control. In addition, $20 \mu \mathrm{L}$ of $\mathrm{MH}$ broth was served as broth control, [Frame1] and $20 \mu \mathrm{L}$ of $\mathrm{MH}$ broth containing $0.5 \%$ ethanol was used as ethanol control. The 96-well plate was covered by a low evaporation lid and incubated at $30^{\circ} \mathrm{C}$ with aeration $(169 \mathrm{rpm})$. The bioluminescence signal of each well was measured after $4.5 \mathrm{~h}$ incubation using a Tecan microplate reader (Infinite 200 Pro; Tecan Life Sciences). The bioluminescence signals of ethanol control were subtracted from the treatment groups as baselines, and the signals of broth control were subtracted from non-treated groups.

\section{Biofilm Cultivation}

C. jejuni biofilm was cultivated according to the protocol described in a previous study with some modifications (Müsken et al., 2010). Three C. jejuni strains were separately cultivated in $\mathrm{MH}$ broth for $16 \mathrm{~h}$ and then diluted to $\sim 2 \times 10^{8} \mathrm{CFU} / \mathrm{mL}$. One hundred microliters of $C$. jejuni culture were added to each well of a sterile polystyrene 96-well plate, followed by the addition of $100 \mu \mathrm{L}$ of decanoic acid and lauric acid working solutions, respectively. $\mathrm{MH}$ broth and $\mathrm{MH}$ broth with $0.5 \%$ ethanol were separately served as broth control and ethanol control. The plates were covered with low evaporation lids and incubated at $37^{\circ} \mathrm{C}$ under the microaerobic condition for $72 \mathrm{~h}$.

\section{Quorum Sensing Inhibitory Effect Effect of Fatty Acids on Campylobacter jejuni Al-2 Activity During Biofilm Formation}

To determine the quorum sensing inhibitory effect of both decanoic acid and lauric acid, the supernatant in each well of the 96-well plate for biofilm cultivation was separately collected after 72-h incubation. The collected supernatant was centrifuged at $8,000 \times g$ for $10 \mathrm{~min}$ and passed through $0.22-\mu \mathrm{m}$ PES filters. CFSs were stored at $-20^{\circ} \mathrm{C}$ until further use.

The AI-2 activity in the supernatant during biofilm formation was then determined using $V$. harveyi AI-2 assay as aforementioned in 2.3. In addition, $\mathrm{MH}$ broth $(20 \mu \mathrm{L})$ was served as broth control, and $\mathrm{MH}$ broth containing $0.5 \%$ ethanol $(20 \mu \mathrm{L})$ was used as ethanol control. The signals of broth control were subtracted from non-treated groups, and the bioluminescence signals of ethanol control were subtracted from the treatment groups as baselines.

\section{Effect of Fatty Acid on Al-2 Activity of Campylobacter jejuni Cell-Free Supernatants}

To determine whether there is a direct interaction between C. jejuni AI-2 signaling molecules and fatty acids, C. jejuni was grown in $\mathrm{MH}$ broth overnight at $37^{\circ} \mathrm{C}$ under the microaerobic condition. Cell-free supernatants were collected by centrifugation and filtration as aforementioned. One hundred microliters of the collected cell-free supernatants were separately mixed with $100 \mu \mathrm{L}$ of 200, 100, and $50 \mathrm{ppm}$ decanoic acid and lauric acid working solutions and incubated for $1 \mathrm{~h}$ at $37^{\circ} \mathrm{C}$. AI-2 activity of each treated cell-free supernatant was determined using V. harveyi AI-2 assay.

\section{Quantification of Campylobacter jejuni Biofilm Biomass}

Crystal violet staining assay was used to quantify the total biomass of formed C. jejuni biofilms according to the protocol described in a previous study with modifications (Feng et al., 2018). Each well of the polystyrene plate was washed twice using PBS and air-dried for $30 \mathrm{~min}$. One hundred and fifty microliters of $1 \%(\mathrm{w} / \mathrm{v})$ crystal violet solution was added into each well to stain the attached biofilm for $15 \mathrm{~min}$. Then, the crystal violet solution was removed from each well, followed by washing three times with sterile deionized water to remove any unbounded staining residuals. The plates were air-dried for $30 \mathrm{~min}$, followed by the addition of $200 \mu \mathrm{L}$ of $95 \%$ ethanol (v/v) to dissolve the biofilm-associated crystal violet for $10 \mathrm{~min}$. The absorbance level of the dissolved dye in each well was determined using a microplate reader at $595 \mathrm{~nm}$ (Infinite 200 Pro; Tecan Life Sciences). Sterile $\mathrm{MH}$ broth and $\mathrm{MH}$ broth with each fatty acid were stained to serve as broth control and treatment controls.

TABLE 2 | The highest subinhibitory concentrations of the selected compounds against three $C$. jejuni isolates.

\begin{tabular}{|c|c|c|}
\hline Compound & Strain & $S I C(p p m)$ \\
\hline \multirow[t]{3}{*}{ Hexanoic acid } & F38011 & \multirow{3}{*}{$\begin{array}{c}\text { No inhibition at } \\
\text { conc. } \leq 200\end{array}$} \\
\hline & 11168 & \\
\hline & 33560 & \\
\hline \multirow[t]{3}{*}{ Octanoic acid } & F38011 & \multirow{3}{*}{$\begin{array}{c}\text { No inhibition at } \\
\text { conc. } \leq 200 \\
150\end{array}$} \\
\hline & 11168 & \\
\hline & 33560 & \\
\hline \multirow[t]{3}{*}{ Decanoic acid } & F38011 & \multirow[t]{3}{*}{150} \\
\hline & 11168 & \\
\hline & 33560 & \\
\hline \multirow[t]{3}{*}{ Lauric acid } & F38011 & \multirow[t]{3}{*}{100} \\
\hline & 11168 & \\
\hline & 33560 & \\
\hline \multirow[t]{3}{*}{ 4-Hydroxy-2,5-dimethyl-3(2H)-furanone } & F38011 & \multirow{3}{*}{$\begin{array}{c}\text { No inhibition at } \\
\text { conc. } \leq 200\end{array}$} \\
\hline & 11168 & \\
\hline & 33560 & \\
\hline \multirow[t]{3}{*}{ 5-Ethyl-4-hydroxy-2-methyl-2(2H)-furanone } & F38011 & \multirow{3}{*}{$\begin{array}{c}\text { No inhibition at } \\
\text { conc. } \leq 200\end{array}$} \\
\hline & 11168 & \\
\hline & 33560 & \\
\hline \multirow[t]{3}{*}{ 2,5-Dimethyl-3-oxo-2(H)-fur-4-yl butyrate } & F38011 & \multirow{3}{*}{$\begin{array}{c}\text { No inhibition at } \\
\text { conc. } \leq 200\end{array}$} \\
\hline & 11168 & \\
\hline & 33560 & \\
\hline \multirow[t]{3}{*}{ Crotonic acid } & F38011 & \multirow{3}{*}{$\begin{array}{c}\text { No inhibition at } \\
\text { conc. } \leq 200\end{array}$} \\
\hline & 11168 & \\
\hline & 33560 & \\
\hline \multirow[t]{3}{*}{ Trans-Ferulic acid } & F38011 & \multirow{3}{*}{$\begin{array}{c}\text { No inhibition at } \\
\text { conc. } \leq 200\end{array}$} \\
\hline & 11168 & \\
\hline & 33560 & \\
\hline \multirow[t]{3}{*}{ Quercetin } & F38011 & 25 \\
\hline & 11168 & 50 \\
\hline & 33560 & 25 \\
\hline \multirow[t]{3}{*}{ Trans-cinnamaldehyde } & F38011 & 12.5 \\
\hline & 11168 & \\
\hline & 33560 & \\
\hline \multirow[t]{3}{*}{ Naringenin } & F38011 & 50 \\
\hline & 11168 & 25 \\
\hline & 33560 & 25 \\
\hline
\end{tabular}


The broth control and treatment controls were subtracted from the non-treated and treated groups, respectively, as the baselines.

\section{Bacterial Motility Assay}

The effect of fatty acids on C. jejuni cell motility was assessed using the soft agar plate assay (Kalmokoff et al., 2006). Soft agar plates contained $25 \mathrm{~mL}$ of $\mathrm{MH}$ broth supplemented with $0.4 \%$ agar with or without the addition of decanoic acid and lauric acid. Soft agar plates that contained $0.5 \%$ ethanol were used as the negative control. C. jejuni overnight culture was adjusted to $\sim 10^{8} \mathrm{CFU} / \mathrm{mL}$, and $2 \mu \mathrm{L}$ of the adjusted culture was stab inoculated at the center of the soft agar plates. The inoculated plates were then incubated at $37^{\circ} \mathrm{C}$ for $48 \mathrm{~h}$ under the microaerobic condition. The motility of $C$. jejuni cells was determined by measuring the distance of bacterial migrating from the inoculation site.

\section{Statistical Analysis}

All experiments were conducted at least in three biological replicates. Results were presented as the mean \pm standard deviation. Data analysis and visualization were performed using Origin (version OriginPro 2020, OriginLab Corporation, United States). One-way ANOVA followed by appropriate post hoc test was used to determine if the difference was statistically significant $(P<0.05)$.

\section{RESULTS AND DISCUSSION}

\section{Subinhibitory Concentrations of Fatty Acids}

Subinhibitory concentration of an antimicrobial is the concentration below the minimum inhibitory concentration of that compound. Exposing microorganisms to the subinhibitory concentration of an antimicrobial allows the observation of its effects on cellular processes without disturbing cell viability. Quorum sensing is a cell-to-cell communication process underpinned by various biological processes (Papenfort and Bassler, 2016). Using subinhibitory concentration to study quorum sensing quenching effect can largely avoid bias caused by bactericidal effect.

The highest subinhibitory concentrations of the tested compounds against four C. jejuni isolates are shown in Table 2. Among 12 tested compounds, hexanoic acid, 4-hydroxy-2,5-dimethyl-3(2H)-furanone, 5-ethyl-4-hydroxy2-methyl-2(2H)-furanone, 2,5-dimethyl-3-oxo-2(H)-fur-4-yl butyrate, crotonic acid, and trans-ferulic acid did not show growth inhibitory effect on all four strains at the concentration of 200 ppm, which was the highest tested concentration. For the four fatty acids (i.e., hexanoic acid, octanoic acid, decanoic acid, and lauric acid), the inhibitory concentrations decreased along with the increase of alkyl chain length. Susceptibility of the three tested C. jejuni strains to octanoic acid, lauric acid, quercetin, and naringenin was slightly different. For example, C. jejuni ATCC 33560 was more susceptible to octanoic acid than the other tested strains.

\section{Selection of Al-2 Inhibitors}

Twelve compounds were screened and assessed for their ability to inhibit AI-2 activities of C. jejuni ATCC 33560 at the subinhibitory concentrations, as shown in Table 1. The inhibitory effects of AI-2 mediated quorum sensing activity in C. jejuni by the compounds were determined as the intensity of bioluminescence signal emitted by the reporter strain $V$. harveyi BB170. Among the 12 compounds, both decanoic acid and lauric acid significantly inhibited AI-2 activities of C. jejuni,

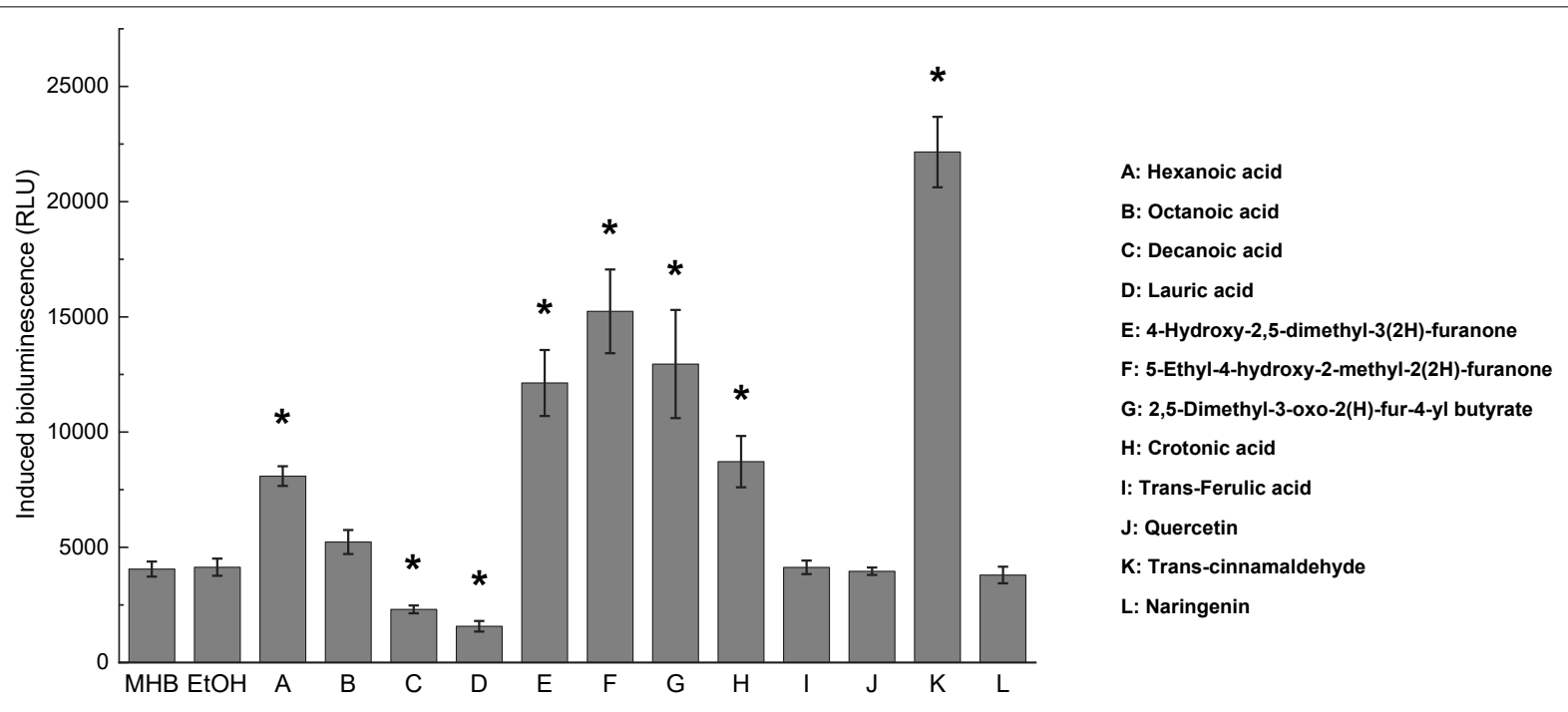

FIGURE 1 | Screening of $C$. jejuni autoinducer-2 inhibitor. Bioluminescence activity of $C$. jejuni ATCC 33560 treated with 12 different compounds was compared to the ethanol control $(\mathrm{EtOH})$. MH broth containing $0.5 \%$ ethanol was used as ethanol control. Each bar represents the average of three biological replicates with standard deviation. MHB was serve as broth control and EtOH was negative control. * Statistical significance between negative control and treated samples was determined by one-way ANOVA coupled with Dunnett's multiple comparisons test $(P<0.05$ was considered as statistically significant). 

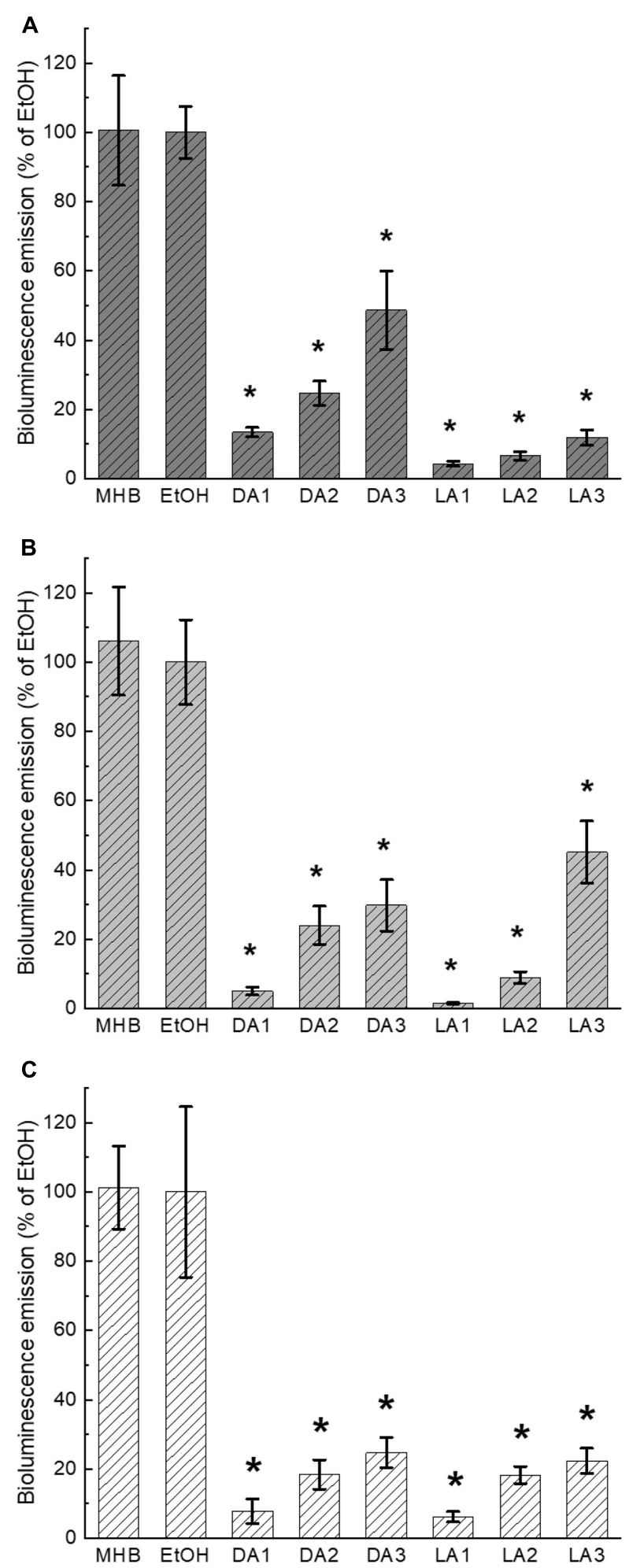

MHB: $\mathrm{MH}$ broth

EtOH: $\mathrm{MHB}+0.5 \% \mathrm{EtOH}$

DA1: DA at $100 \mathrm{ppm}$

DA2: DA at $50 \mathrm{ppm}$

DA3: DA at $25 \mathrm{ppm}$

LA1: LA at $100 \mathrm{ppm}$

LA2: LA at $50 \mathrm{ppm}$

LA3: LA at $25 \mathrm{ppm}$

FIGURE 2 | Inhibition of C. jejuni Al-2 activity by decanoic acid (DA) and lauric acid (LA). C. jejuni strain F38011 (A), NCTC11168 (B), ATCC33560 (C).

Bioluminescence emission of $V$. harveyi BB170 with the addition of cell-free supernatants collected from the supernatant of $C$. jejuni biofilm incubated with or without fatty acids for $72 \mathrm{~h}$ at $37^{\circ} \mathrm{C}$ under microaerobic condition was separately determined. $\mathrm{MH}$ broth containing $0.5 \%$ ethanol was used as ethanol control. MHB is the broth control and $\mathrm{EtOH}$ is the negative control. Bioluminescence level of the negative control was set at $100 \%$. Bioluminescence emission of each treatment was normalized to the control. Three biological replicates were performed. * Statistical significance between negative control and treated samples was determined by one-way ANOVA coupled with Dunnett's multiple comparisons test $(P<0.05$ was considered statistically significant). 

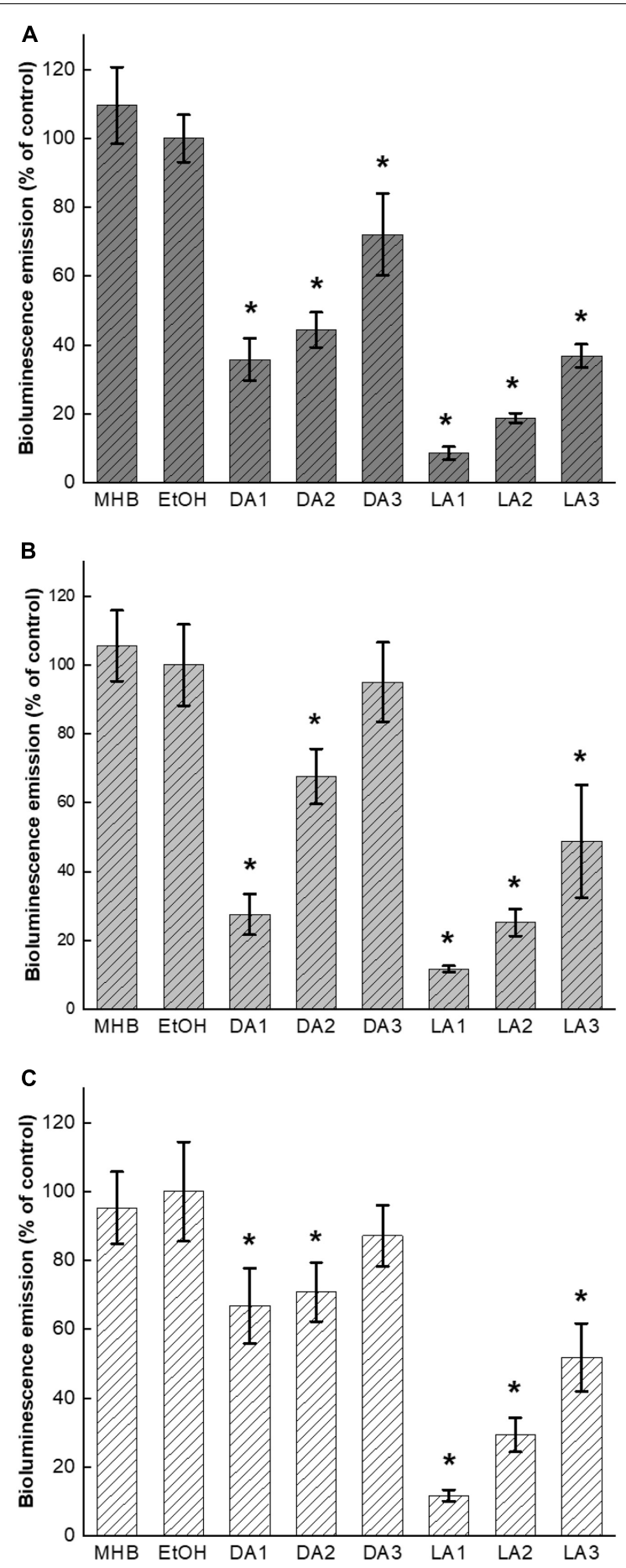

MHB: MH broth

$\mathrm{EtOH}: \mathrm{MHB}+0.5 \% \mathrm{EtOH}$

DA1: DA at $100 \mathrm{ppm}$

DA2: DA at $50 \mathrm{ppm}$

DA3: DA at $25 \mathrm{ppm}$

LA1: LA at $100 \mathrm{ppm}$

LA2: LA at $50 \mathrm{ppm}$

LA3: LA at $25 \mathrm{ppm}$

FIGURE 3 | Influence of decanoic acid (DA) and lauric acid (L.A.) on Al-2 activity in C. jejuni cell-free supernatants. C. jejuni strain F38011 (A), NCTC11168 (B), and ATCC33560 (C). Cell-free supernatant from each $\mathrm{C}$. jejuni culture grown in $\mathrm{MH}$ broth was incubated with fatty acids for $1 \mathrm{~h}$, respectively. $\mathrm{MH}$ broth containing $0.5 \%$ ethanol was used as ethanol control. $\mathrm{MHB}$ is the broth control and $\mathrm{EtOH}$ is the negative control. Bioluminescence level of the negative control was set at $100 \%$.

Bioluminescence emission of each treatment was normalized to the control. Three biological replicates were performed. * Statistical significance between negative control and treated samples was determined by one-way ANOVA coupled with Dunnett's multiple comparisons test $(P<0.05$ was considered statistically significant).

whereas hexanoic acid, three furanones, crotonic acid, and transcinnamaldehyde increased the AI-2-induced bioluminescence (Figure 1). Octanoic acid, trans-ferulic acid, quercetin, and naringenin had no significant effect on AI-2 activity of C. jejuni $(P>0.05)$. Therefore, decanoic acid and lauric acid might be able to quench AI-2 mediated quorum sensing activities 
in C. jejuni. In addition, there was no significant difference in bioluminescence activities between $\mathrm{MH}$ broth control and ethanol control $(P>0.05)$. Thus, the effect of carrier ethanol on C. jejuni AI-2 activity at $0.5 \%$ was negligible.

\section{Quorum Sensing Inhibitory Effect of Decanoic Acid and Lauric Acid Quorum Sensing Inhibitory Effect During Campylobacter jejuni Biofilm Formation}

AI-2 activities within the supernatant of biofilms of all three C. jejuni strains were significantly reduced $(P<0.05)$ by both decanoic acid and lauric acid at all the tested subinhibitory concentrations (100 ppm, $50 \mathrm{ppm}, 25 \mathrm{ppm}$ ) compared to the ethanol control (Figure 2). There was no significant difference in AI-2 activity between $\mathrm{MH}$ broth control and ethanol control, indicating that $0.5 \%$ ethanol added in the broth as the carrier solvent did not affect C. jejuni AI-2 activity. The addition of different concentrations of either decanoic acid or lauric acid demonstrated a concentration-dependent inhibitory effect on C. jejuni AI-2 activity. Lauric acid at 100 ppm showed the most substantial quorum quenching effect on all C. jejuni strains used in this study. However, the inhibitory effects of decanoic acid and lauric acid varied among different $C$. jejuni strains. For example, AI-2 activity of C. jejuni $\mathrm{F} 38011$ was reduced $>80 \%$ by lauric acid at all three concentrations after 3-day incubation (Figure 2A). In comparison, lauric acid at 25 ppm only decreased $65 \%$ of AI-2 activity of C. jejuni NCTC11168 (Figure 2B).

\section{Influence of Fatty Acids on Al-2 Activity of Campylobacter jejuni Cell-Free Supernatants}

To investigate whether there is a direct interaction between fatty acids and C. jejuni AI-2 molecules, cell-free supernatants collected from C. jejuni overnight culture were directly incubated with decanoic acid and lauric acid at the subinhibitory concentrations, $100 \mathrm{ppm}, 50 \mathrm{ppm}, 25 \mathrm{ppm}$, respectively. The inhibitory effect was significant $(P<0.05)$ in all the treatment groups except for decanoic acid at $25 \mathrm{ppm}$ on C. jejuni NCTC11168 and ATCC33560 (Figure 3). The results of direct interaction were similar to those shown in Figure 2. Concentration-dependent inhibitory effect of decanoic acid and lauric acid was observed. Lauric acid at $100 \mathrm{ppm}$ showed the most potent inhibition against $C$. jejuni AI-2 activity.

Quorum sensing circuits involve a range of enzymes and receptors so that there are multiple potential targets for quorum quenching. Production of signaling molecules can be inhibited by repression/inactivation of enzymes required for A.I.s synthesis or by inactivation of the AI molecules. Besides, quorum sensing can be inhibited at the perception stage by blocking the receptors (Hentzer and Givskov, 2003). A previous study reported that several long-chain fatty acids identified from poultry meat wash were able to reduce the activity of in vitro synthesized AI-2. The authors speculated that the inhibition could be caused by either direct interaction with AI-2 molecules or interference of AI-2 receptor (Widmer et al., 2007). In the current study, inhibition of AI-2 activity was observed in both circumstances, namely mixing fatty acids with cell-free supernatants and treating C. jejuni cells. Our preliminary experiment confirmed that treatment of decanoic acid and lauric acid at $200 \mathrm{ppm}$ did not affect the viability of the $V$. harveyi $\mathrm{BB} 170$ reporter strain. Thus, we speculated that interaction between decanoic acid and lauric acid with the signaling molecules or the receptor resulted in the loss of AI-2 activities.

\section{Effect of Fatty Acids on Campylobacter jejuni Biofilm Biomass}

The effects of decanoic acid and lauric acid at different subinhibitory concentrations on C. jejuni biofilm formation are shown in Figure 4. There was a variation between inhibitory effects on quorum sensing and biofilm formation. Although both decanoic acid and lauric acid were potent in inhibiting AI-2 activity of C. jejuni, they were not effective in inhibiting C. jejuni biofilm formation at low concentrations. Among the three tested C. jejuni strains, decanoic acid at $100 \mathrm{ppm}$ reduced the total biofilm biomass of C. jejuni F38011 and ATCC33560 by 35 and $10 \%$, respectively (Figures 4A,C). Lauric acid at $100 \mathrm{ppm}$ reduced biofilm formation of all three C. jejuni strains, ranging from 50\% for NCTC11168 to 10\% for F38011 and ATCC33560. However, a stimulating effect on C. jejuni NCTC11168 biofilm formation was observed at the treatment groups of 50 and 25 ppm decanoic acid and 25 ppm lauric acid.

C. jejuni biofilm formation has been recognized as one of AI2 mediated bacterial social behaviors (Plummer, 2012). Reeser et al. (2007) reported that biofilm formation level was lower in luxS-deficient C. jejuni mutant than that of wildtype, and biofilm formation ability of the mutant was partially restored by adding AI-2 containing C. jejuni cell-free supernatants. However, another study indicated that phenotypes of luxSdeficient C. jejuni mutants varied along with the changes of mutagenesis, strains, and cultivation conditions (Adler et al., 2014). Besides, biofilm formation capabilities of various C. jejuni strains are different due to the variations in genetic features (Gunther and Chen, 2009). Because of the potential differences, we used three C. jejuni isolates from different origins to ensure the results were representative. Decanoic acid and lauric acid effectively reduced biofilm formation of C. jejuni F38011, but not NCTC11168, regardless of their evident inhibitory effect of AI2 activity on this strain. However, biofilm formation is a highly complex and dynamic process. Multifaceted mechanisms, such as modulation of non-coding RNAs and transcriptional regulators, also involved in the orchestration of biofilm formation, are regulated via quorum sensing (Goo et al., 2015). Moreover, variation in growth rate of bacteria, formation of microcolonies at early biofilm formation stage, and threshold of detection of quorum sensing molecules can also lead to the difference in responding to AI-2 changes (Hense et al., 2007). Taken together, the relationship between inhibition of quorum sensing and reduction of biofilm formation is not straightforward.

\section{Effect of Fatty Acids on Campylobacter jejuni Cell Motility}

Flagella-driven motility has been validated to be one of the virulence factors of $C$. jejuni because it is critical for its 

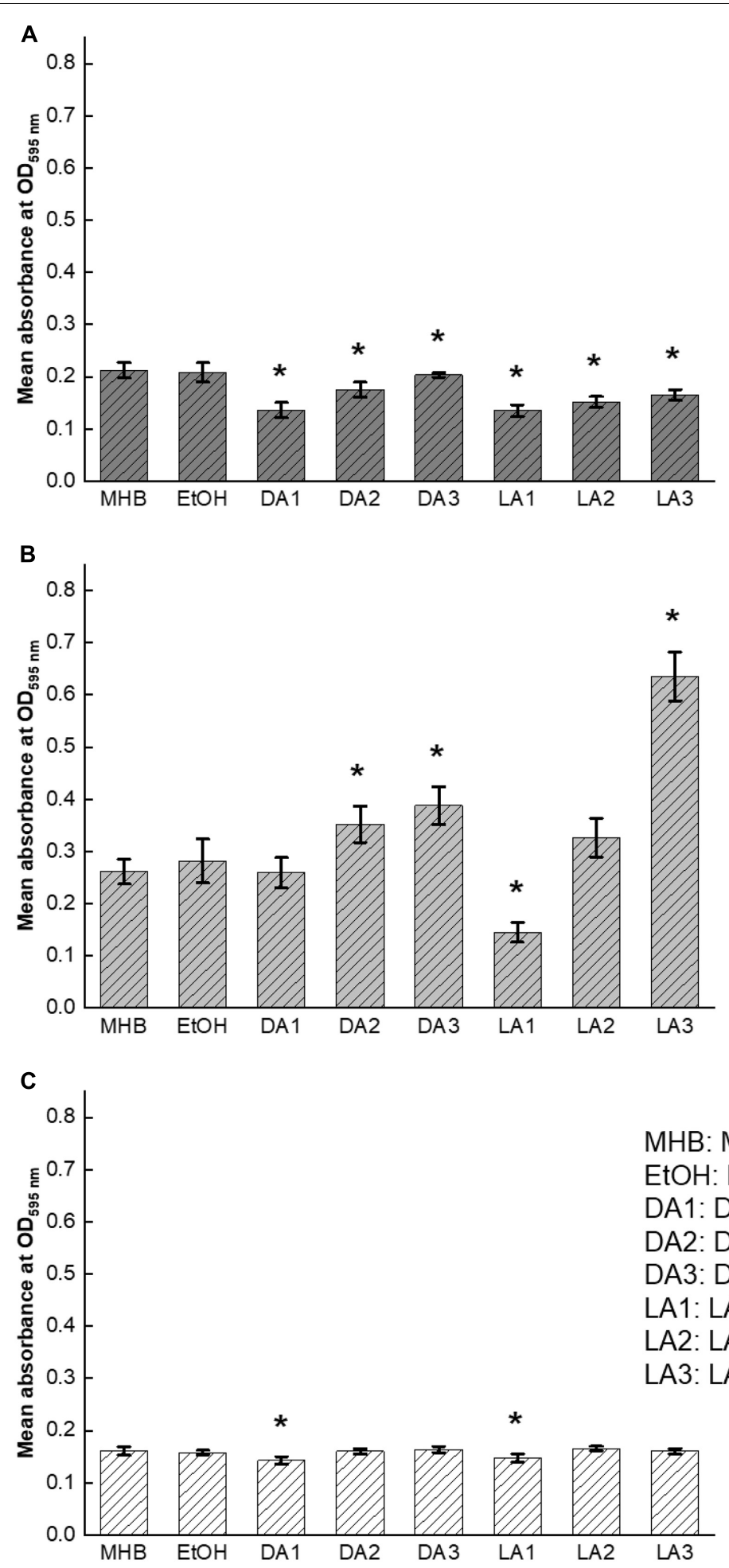

FIGURE 4 | Effect of decanoic acid (DA) and lauric acid (L.A.) on biofilm formation of C. jejuni. C. jejuni strain F38011 (A), NCTC11168 (B), and ATCC33560 (C). Total biomass of the formed biofilms was quantified by crystal violet staining at $\mathrm{OD}_{595 \mathrm{~nm}}$ after $72 \mathrm{~h}$ incubation with or without fatty acids at $37^{\circ} \mathrm{C}$ under microaerobic condition. $\mathrm{MH}$ broth containing $0.5 \%$ ethanol was used as ethanol control. $\mathrm{MH}$ broth and $\mathrm{MH}$ broth containing $0.5 \% \mathrm{EtOH}$ are served as the broth control and negative control, respectively. Three biological replicates were performed. * Statistical significance between negative control and treated samples was determined by one-way ANOVA coupled with Dunnett's multiple comparisons test $(P<0.05$ was considered as statistically significant). 


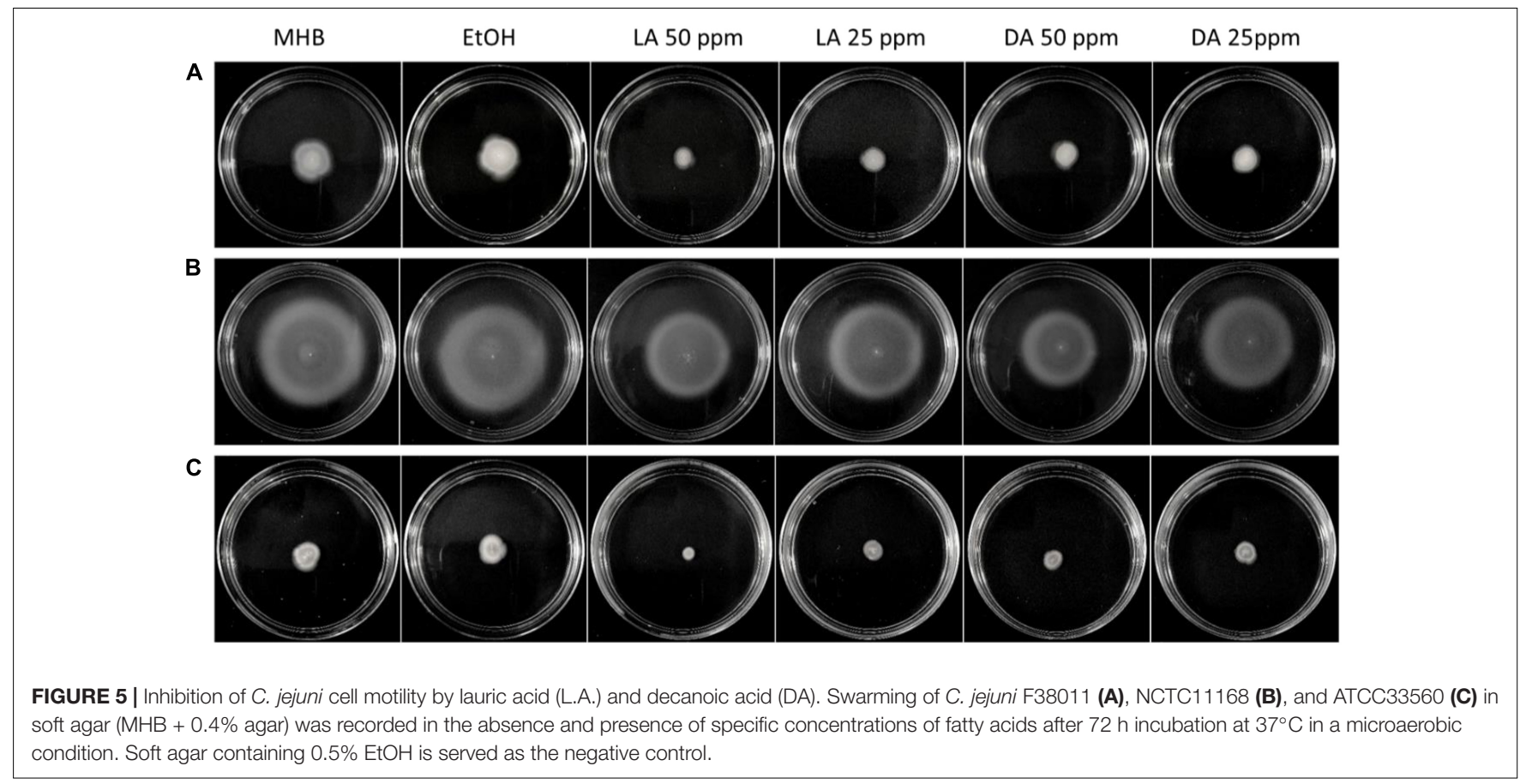

colonization (Plummer, 2012). This bacterial process has been associated with AI-2 mediated quorum sensing and plays an important role in biofilm formation (Brackman et al., 2011). C. jejuni motility levels varied among different strains. C. jejuni NCTC11168 was higher motile than F38011 and ATCC33560 while C. jejuni NCTC11168 showed the largest migration distance (Figure 5). Interestingly, this strain also showed the highest level of biofilm formation among the three tested strains (Figure 4). C. jejuni motility mediated by AI-2 was associated with the development of biofilm structure and survival of C. jejuni. We evaluated the effects of decanoic acid and lauric acid on C. jejuni motility and identified that both decanoic acid and lauric acid were able to reduce the motility of all tested strains (Figure 5). Inhibition of C. jejuni migration was more evident at a high concentration of fatty acid treatment than those low concentration groups.

\section{CONCLUSION}

Both decanoic acid and lauric acid at subinhibitory concentrations effectively reduced the AI-2 activity of C. jejuni by over $50 \%$ after $72-\mathrm{h}$ incubation. Direct addition of either decanoic acid or lauric acid to C. jejuni cell-free supernatants also decreased AI-2 activity in a concentration-dependent manner. Both fatty acids reduced C. jejuni F38011 biofilm formation at all tested concentrations, whereas reduction of biofilm formation of C. jejuni ATCC33560 was only achieved by decanoic acid and lauric acid at $100 \mathrm{ppm}$. Motility of all tested C. jejuni strains was impaired by both fatty acids. The effect of fatty acids on C. jejuni biofilm formation might be determined by a complex mechanism of action. Our current results provide new insights into the use of fatty acids for C. jejuni control in agri-food and clinical settings.

\section{DATA AVAILABILITY STATEMENT}

The original contributions presented in the study are included in the article/Supplementary Material, further inquiries can be directed to the corresponding author/s.

\section{AUTHOR CONTRIBUTIONS}

SL and XL developed this project. SL and GG conceptualized and designed the experiments. SL, KC, and $\mathrm{MH}$ performed the experiment. SL analyzed the data. SL, MH, and XL drafted the manuscript. All authors contributed to the article and approved the submitted version.

\section{FUNDING}

This work was supported by the Natural Sciences and Engineering Research Council of Canada to XL in the form of a Discovery Grant from the Natural Sciences and Engineering Research Council of Canada (NSERC RGPIN-2019-03960) and a Discovery Accelerator Grant (NSERC RGPIN-2019-00024).

\section{SUPPLEMENTARY MATERIAL}

The Supplementary Material for this article can be found online at: https://www.frontiersin.org/articles/10.3389/fmicb.2021. 811506/full\#supplementary-material 


\section{REFERENCES}

Adler, L., Alter, T., Sharbati, S., and Gölz, G. (2014). Phenotypes of campylobacter jejuni luxS mutants are depending on strain background, kind of mutation and experimental conditions. PLoS One 9:e104399. doi: 10.1371/journal.pone. 0104399

Barbieri, R., Coppo, E., Marchese, A., Daglia, M., Sobarzo-Sánchez, E., Nabavi, S. F., et al. (2017). Phytochemicals for human disease: an update on plantderived compounds antibacterial activity. Microbiol. Res. 196, 44-68. doi: 10. 1016/j.micres.2016.12.003

Bassler, B. L., Greenberg, E. P., and Stevens, A. M. (1997). Cross-species induction of luminescence in the quorum-sensing bacterium Vibrio harveyi. J. Bacteriol. 179, 4043-4045. doi: 10.1128/jb.179.12.4043-4045.1997

Bettenworth, V., Steinfeld, B., Duin, H., Petersen, K., Streit, W. R., Bischofs, I., et al. (2019). Phenotypic heterogeneity in bacterial quorum sensing systems. J. Mol. Biol. 431, 4530-4546. doi: 10.1016/j.jmb.2019.04.036

Brackman, G., Cos, P., Maes, L., Nelis, H. J., and Coenye, T. (2011). Quorum sensing inhibitors increase the susceptibility of bacterial biofilms to antibiotics in vitro and in vivo. Antimicrob. Agents Chemother. 55, 2655-2661. doi: 10. 1128/AAC.00045-11

Centers for Disease Control Prevention (2019). Antibiotic Resistance Threats In The United States. Atlanta, GA: US Department of Health and Human Services.

Duarte, A., Luís, Â, Oleastro, M., and Domingues, F. C. (2016). Antioxidant properties of coriander essential oil and linalool and their potential to control Campylobacter spp. Food Control 61, 115-122. doi: 10.1016/j.foodcont.2015.0 9.033

Feng, J., Ma, L., Nie, J., Konkel, M. E., and Lu, X. (2018). Environmental stress-induced bacterial lysis and extracellular DNA release contribute to Campylobacter jejuni biofilm formation. Appl. Environ. Microbiol. 84, e02068e02117. doi: 10.1128/AEM.02068-17

García-Sánchez, L., Melero, B., and Rovira, J. (2018). Campylobacter In The Food Chain, In Advances In Food And Nutrition Research. Amsterdam: Elsevier, 215-252.

Goo, E., An, J. H., Kang, Y., and Hwang, I. (2015). Control of bacterial metabolism by quorum sensing. Trends Microbiol. 23, 567-576. doi: 10.1016/j.tim.2015.0 5.007

Greenberg, E., Hastings, J. W., and Ulitzur, S. (1979). Induction of luciferase synthesis in Beneckea harveyi by other marine bacteria. Arch. Microbiol. 120, 87-91. doi: 10.1016/j.bbaexp.2006.05.005

Gunther, I. V. N. W., and Chen, C.-Y. (2009). The biofilm forming potential of bacterial species in the genus Campylobacter. Food Microbiol. 26, 44-51. doi: 10.1016/j.fm.2008.07.012

Guo, X., Stedtfeld, R. D., Hedman, H., Eisenberg, J. N., Trueba, G., Yin, D., et al. (2018). Antibiotic resistome associated with small-scale poultry production in rural Ecuador. Environ. Sci. Technol. 52, 8165-8172. doi: 10.1021/acs.est. $8 \mathrm{~b} 01667$

Hense, B. A., Kuttler, C., Müller, J., Rothballer, M., Hartmann, A., and Kreft, J.U. (2007). Does efficiency sensing unify diffusion and quorum sensing? Nature Rev. Microbiol. 5, 230-239. doi: 10.1038/nrmicro1600

Hentzer, M., and Givskov, M. (2003). Pharmacological inhibition of quorum sensing for the treatment of chronic bacterial infections. J. Clin. Invest. 112, 1300-1307. doi: 10.1172/JCI20074

Jeon, B., Itoh, K., Misawa, N., and Ryu, S. (2003). Effects of quorum sensing on flaA transcription and autoagglutination in Campylobacter jejuni. Microbiol. Immunol. 47, 833-839. doi: 10.1111/j.1348-0421.2003.tb03 449.x

Jiang, Q., Chen, J., Yang, C., Yin, Y., and Yao, K. (2019). Quorum sensing: a prospective therapeutic target for bacterial diseases. BioMed. Res. Int. 2019:2015978. doi: 10.1155/2019/2015978

Jiang, T., and Li, M. (2013). Quorum sensing inhibitors: a patent review. Expert Opin. Ther. Pat. 23, 867-894. doi: 10.1517/13543776.2013.77 9674

Kaakoush, N. O., Castaño-Rodríguez, N., Mitchell, H. M., and Man, S. M. (2015). Global epidemiology of Campylobacter infection. Clin. Microbiol. Rev. 28, 687-720. doi: $10.1128 / \mathrm{cmr} .00006-15$
Kalmokoff, M., Lanthier, P., Tremblay, T.-L., Foss, M., Lau, P. C., Sanders, G., et al. (2006). Proteomic analysis of Campylobacter jejuni 11168 biofilms reveals a role for the motility complex in biofilm formation. J. Bacteriol. 188, 4312-4320. doi: 10.1128/JB.01975-05

Kirk, M. D., Pires, S. M., Black, R. E., Caipo, M., Crump, J. A., Devleesschauwer, B., et al. (2015). World Health Organization estimates of the global and regional disease burden of 22 foodborne bacterial, protozoal, and viral diseases, 2010: a data synthesis. PLoS Med. 12:e1001921. doi: 10.1371/journal.pmed.1001921

Marder, E. P., Cieslak, P. R., Cronquist, A. B., Dunn, J., Lathrop, S., RabatskyEhr, T., et al. (2017). Incidence and trends of infections with pathogens transmitted commonly through food and the effect of increasing use of culture-independent diagnostic tests on surveillance-foodborne diseases active surveillance network, 10 Us Sites, 2013-2016. Morb. Mortal. Wkly Rep. 66:397. doi: 10.15585/mmwr.mm6615al

Marriott, N. G., Gravani, R. B., and Schilling, M. W. (2006). Principles Of Food Sanitation, Vol. 22. New York, NY: Springer.

Martin, M. J., Thottathil, S. E., and Newman, T. B. (2015). Antibiotics Overuse In Animal Agriculture: A Call To Action For Health Care Providers. Washington, D.C: American Public Health Association. doi: 10.2105/AJPH.2015.302870

Mehdi, Y., Létourneau-Montminy, M.-P., Gaucher, M.-L., Chorfi, Y., Suresh, G., Rouissi, T., et al. (2018). Use of antibiotics in broiler production: global impacts and alternatives. Anim. Nutr. 4, 170-178. doi: 10.1016/j.aninu.2018.0 3.002

M'Sadeq, S. A., Wu, S., Swick, R. A., and Choct, M. (2015). Towards the control of necrotic enteritis in broiler chickens with in-feed antibiotics phasing-out worldwide. Anim. Nutr. 1, 1-11. doi: 10.1016/j.aninu.2015.02.004

Müsken, M., Di Fiore, S., Römling, U., and Häussler, S. (2010). A 96-wellplate-based optical method for the quantitative and qualitative evaluation of Pseudomonas aeruginosa biofilm formation and its application to susceptibility testing. Nat. Protoc. 5:1460. doi: 10.1038/nprot.2010.110

Papenfort, K., and Bassler, B. L. (2016). Quorum sensing signal-response systems in Gram-negative bacteria. Nat. Rev. Microbiol. 14:576. doi: 10.1038/nrmicro. 2016.89

Parkhill, J., Wren, B., Mungall, K., Ketley, J., Churcher, C., Basham, D., et al. (2000). The genome sequence of the food-borne pathogen Campylobacter jejuni reveals hypervariable sequences. Nature 403, 665-668. doi: 10.1038/3500 1088

Plummer, P. (2012). LuxS and quorum-sensing in Campylobacter. Front. Cell. Infect. Microbiol. 2:22. doi: 10.3389/fcimb.2012.00022

Plummer, P., Zhu, J., Akiba, M., Pei, D., and Zhang, Q. (2011). Identification of a key amino acid of LuxS involved in AI-2 production in Campylobacter jejuni. PLoS One 6:e15876. doi: 10.1371/journal.pone.0015876

Quiñones, B., Miller, W. G., Bates, A. H., and Mandrell, R. E. (2009). Autoinducer-2 production in Campylobacter jejuni contributes to chicken colonization. Appl. Environ. Microbiol. 75, 281-285. doi: 10.1128/AEM.018 03-08

Reading, N. C., and Sperandio, V. (2006). Quorum sensing: the many languages of bacteria. FEMS Microbiol. Lett. 254, 1-11. doi: 10.1111/j.1574-6968.2005.00 001.x

Reeser, R. J., Medler, R. T., Billington, S. J., Jost, B. H., and Joens, L. A. (2007). Characterization of Campylobacter jejuni biofilms under defined growth conditions. Appl. Environ. Microbiol. 73, 1908-1913. doi: 10.1128/AEM.007 40-06

Sahin, O., Kassem, I. I., Shen, Z., Lin, J., Rajashekara, G., and Zhang, Q. (2015). Campylobacter in poultry: ecology and potential interventions. Avian Dis. 59, 185-200. doi: 10.1637/11072-032315-Review

Schauder, S., Shokat, K., Surette, M. G., and Bassler, B. L. (2001). The LuxS family of bacterial autoinducers: biosynthesis of a novel quorum-sensing signal molecule. Mol. Microbiol. 41, 463-476. doi: 10.1046/j.1365-2958.2001.02 532.x

Schmidt, R. H. (1997). Basic Elements Of Equipment Cleaning And Sanitizing In Food Processing And Handling Operations. Gainesville, FL: University of Florida Cooperative Extension Service, Institute of Food and Agricultural Sciences.

Tack, D. M., Ray, L., Griffin, P. M., Cieslak, P. R., Dunn, J., Rissman, T., et al. (2020). Preliminary incidence and trends of infections with pathogens transmitted 
commonly through food-foodborne diseases active surveillance network, 10 US Sites, 2016-2019. Morb. Mortal. Wkly Rep. 69:509. doi: 10.15585/mmwr. mm6917a1

Tang, Y., Fang, L., Xu, C., and Zhang, Q. (2017). Antibiotic resistance trends and mechanisms in the foodborne pathogen, Campylobacter. Anim. Health Res. Rev. 18, 87-98. doi: 10.1017/S1466252317000135

Us Department of Agriculture Food Safety and Inspection Service (2016). New performance standards for Salmonella and Campylobacter in notready-to-eat comminuted chicken and turkey products and raw chicken parts and changes to related agency verification procedures: response to comments and announcement of implementation schedule. Fed. Regist. 81, 7285-7300.

Wagenaar, J. A., French, N. P., and Havelaar, A. H. (2013). Preventing Campylobacter at the source: why is it so difficult? Clin. Infect. Dis. 57, 16001606. doi: $10.1093 / \mathrm{cid} / \mathrm{cit} 555$

Widmer, K., Soni, K., Hume, M., Beier, R., Jesudhasan, P., and Pillai, S. (2007). Identification of poultry meat-derived fatty acids functioning as quorum sensing signal inhibitors to autoinducer-2 (AI-2). J. Food Sci. 72, M363-M368. doi: $10.1111 / j .1750-3841.2007 .00527 . x$
World Health Organization (2013). The Global View Of Campylobacteriosis: Report Of An Expert Consultation, Utrecht, Netherlands, 9-11 July 2012. Geneva: WHO.

Conflict of Interest: The authors declare that the research was conducted in the absence of any commercial or financial relationships that could be construed as a potential conflict of interest.

Publisher's Note: All claims expressed in this article are solely those of the authors and do not necessarily represent those of their affiliated organizations, or those of the publisher, the editors and the reviewers. Any product that may be evaluated in this article, or claim that may be made by its manufacturer, is not guaranteed or endorsed by the publisher.

Copyright (c) $2022 \mathrm{Li}$, Chan, Hua, Gölz and Lu. This is an open-access article distributed under the terms of the Creative Commons Attribution License (CC BY). The use, distribution or reproduction in other forums is permitted, provided the original author(s) and the copyright owner(s) are credited and that the original publication in this journal is cited, in accordance with accepted academic practice. No use, distribution or reproduction is permitted which does not comply with these terms. 